\title{
Intra-articular platelet-rich plasma vs corticosteroids in the treatment of moderate knee osteoarthritis: a single- center prospective randomized controlled study with a 1-year follow up
}

\author{
Andrejs Elksniņš-Finogejevs ${ }^{1,2^{*}}$, Luis Vidal ${ }^{3}$ and Andrejs Peredistijs ${ }^{2}$
}

\begin{abstract}
Background: Osteoarthritis is the most prevalent type of arthritis, which significantly impacts the patient's mobility and quality of life. Pharmacological treatments for osteoarthritis, such as corticosteroids, produce an immediate reduction of the patient's pain as well as an improvement in the patient's mobility and quality of life, but with a limited long-term efficacy. In this context, platelet-rich plasma (PRP) infiltrations represent a therapeutic tool due to its trophic properties and its ability to control inflammatory processes, especially in musculoskeletal applications. The aim of this study is to evaluate and compare the clinical benefits of PRP when injected intra-articularly vs a commonly used corticosteroid (CS, triamcinolone acetonide, Kenalog ${ }^{\oplus}$ ) in patients affected by mild to moderate symptomatic knee osteoarthritis.
\end{abstract}

Methods: Forty patients affected by symptomatic radiologically confirmed knee osteoarthritis (Kellgren-Lawrence grades II-III) were enrolled in this randomized study. Patients randomized in the PRP group $(n=20)$ received an intra-articular injection of PRP (8 mL) while patients randomized in the CS group $(n=20)$ received an intra-articular injection of triamcinolone acetonide ( $1 \mathrm{~mL}$ of $40 \mathrm{mg} / \mathrm{mL}$ ) plus lidocaine $(5 \mathrm{~mL}$ of $2 \%)$. The pain and function of the target knee were evaluated by the VAS, IKDC, and KSS scales at the baseline (V1), 1 week (V2), 5 weeks (V3), 15 weeks (V4), 30 weeks (V5), and 1 year (V6) after treatment.

(Continued on next page)

\footnotetext{
* Correspondence: andrejs.finogejevs@orto.lv

${ }^{1}$ Faculty of Continuing Education, Rīga Stradiņš University, Riga, Latvia

2"ORTO klinika" Ltd., Riga, Latvia

Full list of author information is available at the end of the article
}

(c) The Author(s). 2020 Open Access This article is licensed under a Creative Commons Attribution 4.0 International License, which permits use, sharing, adaptation, distribution and reproduction in any medium or format, as long as you give appropriate credit to the original author(s) and the source, provide a link to the Creative Commons licence, and indicate if changes were made. The images or other third party material in this article are included in the article's Creative Commons licence, unless indicated otherwise in a credit line to the material. If material is not included in the article's Creative Commons licence and your intended use is not permitted by statutory regulation or exceeds the permitted use, you will need to obtain permission directly from the copyright holder. To view a copy of this licence, visit http://creativecommons.org/licenses/by/4.0/ The Creative Commons Public Domain Dedication waiver (http://creativecommons.org/publicdomain/zero/1.0/) applies to the data made available in this article, unless otherwise stated in a credit line to the data. 
(Continued from previous page)

Results: No serious adverse effects were observed during the follow-up period. A mild synovitis was registered in 15 patients (75\%) in the PRP group within the first week after treatment which resolved spontaneously. Both treatments were effective in relieving pain and improving the knee function in the very short-term follow-up visit (1 week). A high improvement of the subjective scores was observed for both groups up to 5 weeks, with no significative differences between the groups for the VAS, IKDC, or KSS. After 15 weeks of follow-up, the PRP group showed significative improvements in all scores when compared to the CS group. Overall, the patients who received PRP treatment had better outcomes in a longer follow-up visit (up to 1 year) than those who received $C S$.

Conclusions: A single PRP or CS intra-articular injection is safe and improves the short-term scores of pain and the knee function in patients affected by mild to moderate symptomatic knee OA (with no significant differences between the groups). PRP demonstrated a statistically significant improvement over CS in a 1-year follow-up. This study was registered at ISRCTN with the ID ISRCTN46024618.

Keywords: Osteoarthritis, Knee, Platelet-rich plasma, Corticosteroid

\section{Background}

Osteoarthritis (OA) is a slowly progressive chronic disease characterized by pain, loss of function, and deformity of the affected joints. In the past, OA was considered a normal sign of aging and it was described as a degenerative disorder that mainly causes cartilage loss [1]. However, more recent studies have shown that OA occurs and evolves due to the interaction of multiple risk factors affecting the whole joint including the cartilage, subchondral bone, synovium, ligaments, and menisci [2]. Currently, no disease-modifying treatment has been approved, which makes joint replacement the only viable solution for these patients. Non-pharmacological treatments include patient education and self-management, exercises, weight reduction, walking supports (crutches), bracing, shoe and insole modification, local cooling/ heating, acupuncture, and electromagnetic therapy [3, 4]. Pharmacological treatments include topical and oral NSAID [5], intra-articular (IA) injections of corticosteroids, visco-supplements, and blood-derived products, including platelet-rich-plasma (PRP) (highly recommended when the use of oral analgesics or anti-inflammatories fails to release disease symptoms) [6]. The intra-articular (IA) infiltration of corticosteroids provides a short-term reduction in OA pain, and it is considered as an adjunct to core treatment for the relief of moderate to severe pain in people with OA [7]. This approach, however, has limited efficacy in delaying disease progression, as well as undesirable potential side effects when administered in high doses and frequency $[8,9]$. In this context, PRP is proposed as a potential treatment, capable of improving the clinical condition of patients with osteoarthritis [10-15]. A limited number of publications in PRP, in which PRP has been compared to corticosteroid for the treatment of early knee $\mathrm{OA}$, are available in the literature [16-18]. To address these concerns (what concerns, OA or the side effects, not clear), this study was designed to compare the efficacy of a single intra-articular dose of PRP compared to corticosteroids for the treatment of moderate knee OA. The objective of this study is to evaluate the clinical benefits of PRP when injected into the intra-articular space compared to a corticosteroid (triamcinolone acetonide, Kenalog ${ }^{\bullet}$ ), with is a recognized pharmacological treatment in patients with mild to moderate symptomatic knee OA. We hypothesized that intra-articular injection of PRP reduces pain in a very short term (1-week follow-up), similar to triamcinolone acetonide [19], and it leads to an equal or more effective analgesic outcome plus better functional recovery at 1 year follow-up.

\section{Methods \\ Participants}

Demographic variables such as age, sex, body mass index (BMI), and the degree of radiological involvement were collected. A total of 40 patients (32 females and 8 males) with symptomatic, radiologically confirmed, knee OA (Kellgren-Lawrence grades II-III) were enrolled in a prospective, randomized, controlled study in the period from April 2016 to May 2017. The patient's inclusion criteria were over 55 years of age, chronic pain history, swelling, and/or reduced range of motion in the knee joint. Clinical and radiological confirmation of knee's OA (Kellgren-Lawrence grades II-III) were verified by $\mathrm{X}$-ray images in anteroposterior and lateral projections. The patient's exclusion criteria were post-traumatic knee osteoarthritis, pregnancy, breastfeeding, oncological diseases, endocrine diseases (gout, diabetes), autoimmune diseases (rheumatoid arthritis) acute/chronic infectious disease, blood clotting disorders (thrombocytopenia, coagulopathy), previous interventions on the knee joint (i.e., punctures, blockades, arthroscopy), and previous consistent hormonal therapy or non-steroidal antiinflammatory drugs (NSAIDs) treatment (within 10 days prior to the intervention). 


\section{Study design, randomization, and intervention}

This was a single-center prospective randomized controlled study. Potentially eligible patients with knee pain were pre-screened. Patients, who signed an informed consent and met the inclusion criteria, were considered eligible and assigned in a 1:1 ratio into two groups. The patients were randomized using a computer-generated randomized list. Patients assigned to group one (plateletrich plasma (PRP)) received one intra-articular injection of autologous PRP. Patients assigned to group two (corticosteroid (CS)) received one intra-articular injection of corticosteroid. The variation from the pain baseline, measured by the VAS score at 1 year (V1), was considered the primary outcome. The VAS pain score was selfcompleted by the patient. The patient was asked to place a line, perpendicular to the VAS line from the questionnaire, at the point that showed their pain intensity score in their last 7 days of daily activities (walking, working, home activities, house cleaning, and others). Secondary outcomes were the variations in VAS scores, the International Knee Documentation Committee (IKDC 2000 form) score, and the Knee Society Score (KSS) [20] at any time point of the study. All procedures performed in the studies involving human participants were approved by the Latvian local ethics committee and the national health regulatory authority of Latvia. All procedures performed in studies involving human participants were in accordance with the ethical standards of Ethics Committee for Medical and Biomedical Research, Rigas Stradins University (RSU) Ethics Committee, Ref E-9(2), and Riga Eastern Clinical University Hospital Support Foundation. This study was registered at ISRCTN (International Standard Randomized Controlled Trial Number) with the ID ISRCTN46024618, and it was carried out in accordance with the 1964 Declaration of Helsinki. All the patients were informed, before participating in the CT, of the risks of both treatments (including the beneficial and potential adverse effects). Informed consent was obtained from all individual participants included in the study.

\section{PRP preparation method}

PRP was prepared using the Hy-Tissue PRP ${ }^{\circ}$ system, a CE-marked medical device (Fidia, Abano Terme, Italy). To prepare PRP, $18 \mathrm{~mL}$ of peripheral blood was collected and $2 \mathrm{~mL}$ of $3.8 \%$ sodium citrate was added. In order to separate blood components according to their different specific densities, $20 \mathrm{~mL}$ of citrated blood was centrifuged at $1800 \mathrm{rpm}$ for $8 \mathrm{~min}$ using a Duografter $^{\circ}$ II centrifuge (Fidia, Abano Terme, Italy). From this resulting plasmatic fraction, $8 \mathrm{~mL}$ of pure PRP solution was obtained and used for the intraarticular PRP injection.

\section{Infiltration}

Patients in the first group received $8 \mathrm{~mL}$ of an intraarticular infiltration of PRP, and patients in the second group received an intra-articular infiltration of 1 $\mathrm{mL}$ of $40 \mathrm{mg} / \mathrm{mL}$ triamcinolone acetonide (Kenalog ${ }^{\circ}$ ) and $5 \mathrm{~mL}$ of $2 \%$ lidocaine mixed in a single syringe. Arthrocentesis was permitted in both study groups. All the baseline and follow-up visits were performed by an evaluator who was blinded to the treatment throughout the study. The intra-articular knee injection was performed under sterile conditions, without any local or general anesthesia, with a $20-G \times 2.75$ $70 \mathrm{~mm}$ needle using an anterolateral approach. Echographic control (Philips Affinity 70) allowed the correct needle positioning by direct visualization of the $\mathrm{PRP} / \mathrm{CS}$ liquid injected. After this manipulation, an aseptic cool bandage was applied, for $15 \mathrm{~min}$, for local compression. Non-steroidal anti-inflammatory drugs were prohibited for 10 days following the injection. During the follow-up period, patients carried on their ordinary lives without any specific treatments or restrictions.

\section{Statistical analysis}

The sample size calculation used the hypothesis of superiority. The pain was assessed on a visual analog scale (VAS; range $0-10$ points) 12 months after the procedure. An average score of 7.3 was assumed in the control group with a standard deviation of 1.6. This meant that detecting a reduction of 1.5 points in the treatment group vs the control group with a power of $80 \%$ and 2sided significance level of 0.05 would require the inclusion of a total of 36 patients. Considering a possible dropout rate of $10 \%, 40$ patients in total were required (20 patients per group). A difference in the VAS of 1.5 points for the average score and a standard deviation between the 2 groups was fixed (based on published results) [16]. The primary and secondary variables were analyzed using the intention-to-treat principle. Categorical variables were described by percentages and frequencies while continuous variables were described by means, standard deviations, and the 95\% confidence interval of the mean. Parametric tests (unpaired $t$ test) were used for normal distributions and the MannWhitney $U$ test for non-parametric distributions. Data symmetry was analyzed using a D'Agostino and Pearson normality test. Categorical variables were compared using chi-square tests. For all tests, $p<0.05$ was considered statistically significant. Patient randomization was performed using the "Randomizer for Clinical Trial" software. All statistical analyses were performed using GraphPad Prism version 7.00 for Windows (GraphPad Software, La Jolla, CA, USA). 


\section{Results}

A total of 76 patients were screened from April 2016 to May 2018. Of these screened patients, 33 patients did not meet inclusion criteria and 3 declined to participate (Fig. 1). After this, 40 patients were included in either group (per randomization). The mean age of the intention-to-treat patients was $66.5 \pm 8.6$ and $70.1 \pm 9.1$ for the PRP and CS group, respectively. Participants were $84 \%$ men and $16 \%$ women for the PRP group and $71 \%$ and $29 \%$ for the CS group, respectively. There were no significant differences between the 2 groups across all the baseline parameters (Table 1): age, sex, and K-L grade for OA (except for the basal IKDC index ( $p=$ $0.038)$ ). According to the intention-to-treat population, 19 patients were included in the per-protocol evaluation for the PRP group and 17 in the CS group. A total of 4 patients $(10 \%)$ that were randomized (one from the PRP group and three from the CS group) were not included in the analysis. One randomized patient into the PRP group discontinued the evaluation due to the presentation of an autoimmune disorder after 6 months. This patient presented red spots on the face that was later diagnosed with lupus erythematosus. Three patients that were treated with CS were unable to continue the trial due to an arthroplasty at 6,7 , and 9 months.

\section{Clinical results}

Both PRP and CS single injections were effective in reducing pain, and they improved the knee function after the first week of treatment. VAS score changes at 1 year

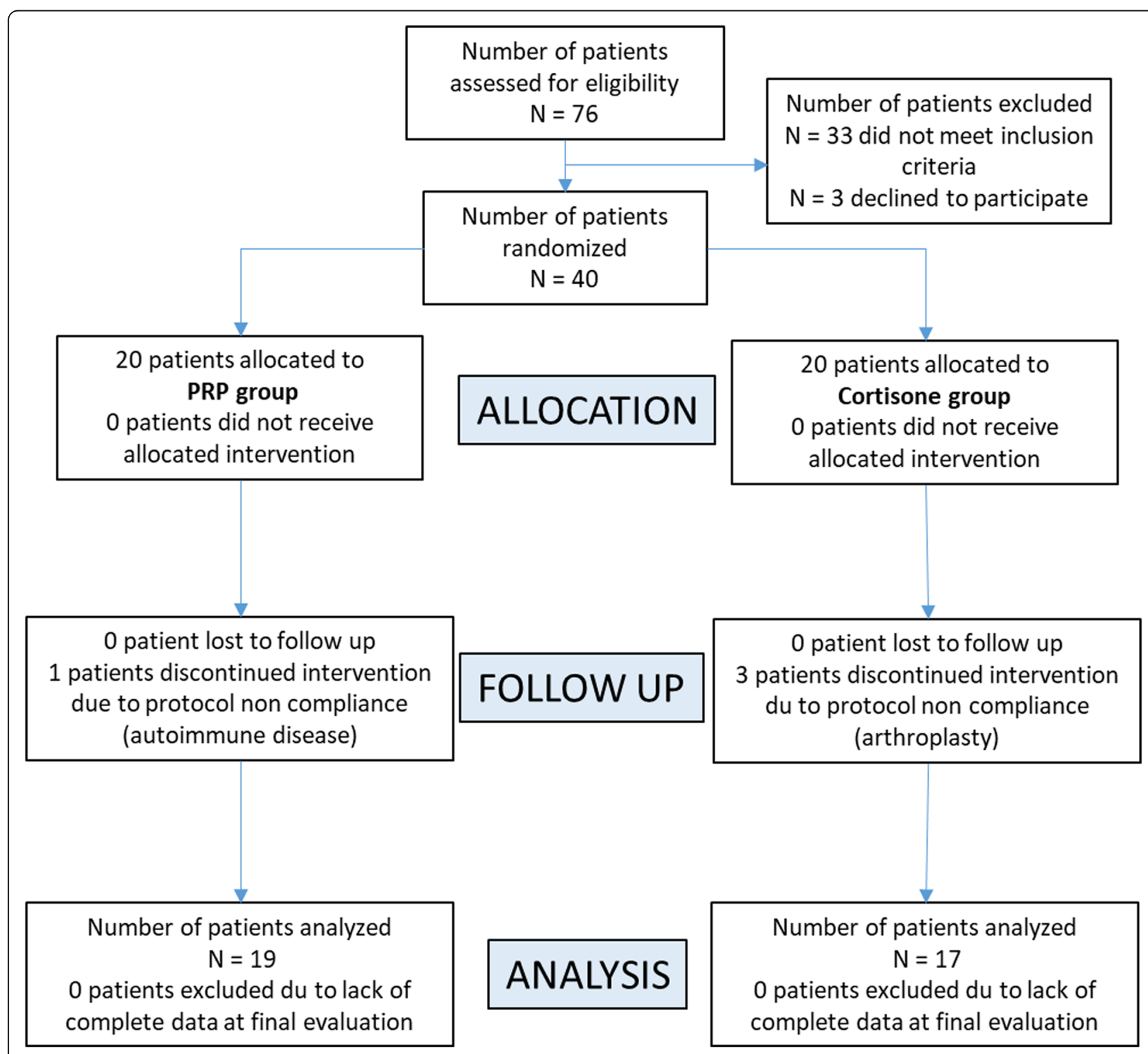

Fig. 1 CONSORT (Consolidated Standards of Reporting Trials) flow diagram for the present study 
Table 1 Baseline characteristics of intent-to-treat patients included in the clinical trial

\begin{tabular}{llll}
\hline & PRP group $(N=20)$ & Corticosteroid group $(n=20)$ & $p$ value \\
\hline Gender, M:F, $n$ & $17: 3$ & $15: 5$ & $\mathrm{~ns}$ \\
Age, years, mean/SD & $66.4 \pm 8.4$ & $70.2 \pm 9.2$ & $\mathrm{~ns}$ \\
BMI, mean/SD & $28.6 \pm 5.0$ & $30.5 \pm 5.8$ & $\mathrm{~ns}$ \\
K-L degree (II/III), $n$ & $5: 15$ & $6: 14$ & $\mathrm{~ns}$ \\
Knee (right/left), $n(\%)$ & $14 / 6(70 \% / 30 \%)$ & $12 / 8(60 \% / 40 \%)$ & $\mathrm{ns}$ \\
VAS baseline, mean/SD & $6.1 \pm 1.2$ & $6.0 \pm 1.4$ & $\mathrm{~ns}$ \\
KSS baseline, mean/SD & $58.3 \pm 7.2$ & $54.0 \pm 8.2$ & $\mathrm{~ns}$ \\
IKDC baseline, mean/SD & $36.6 \pm 10.4$ & $30.0 \pm 8.8$ & 0.0377 \\
\hline
\end{tabular}

Data are provided as mean \pm SD (range), unless indicated otherwise

$B M I$ body mass index, $K-L$, Kellgren-Lawrence classification radiographically confirmed, VAS visual analog scale, KSS Knee Society Score, IKDC International Knee Documentation Committee, ns not significant

(primary clinical outcome) showed a higher mean change from baseline in the PRP group than the CS group (PRP $-3.1 \pm 2.0,-52 \%$; CS $-0.8 \pm 1.8,-14 \%$ ). This difference was significant between groups ( $p=$ 0.0002). The most surprising effect observed was that PRP induced pain relief just as fast as CS. In fact, a significant reduction of pain from baseline for both groups was found 1 week after treatment (mean VAS changePRP - $2.8 \pm 2.3,-47 \%$; CS - 3.4 $\pm 1.2 \%,-58 \% ; p<$ 0.0001). Similarly, significant function improvements from baseline were obtained in the first week for both treatment groups (mean IKDC change-PRP $22.1 \pm$ 16.9, 60\%; CS 35.4 $\pm 10.0,117 \%$ - and mean KSS change-PRP $22.7 \pm 12.3$, 39\%; CS $29.4 \pm 12.8,55 \%$ ). Interestingly, the pain reduction and the knee functional improvement were not significant between both groups in the very short-term follow-up visit (up to 5 weeks; Table 2). The highest change in the VAS score from the baseline was at 3 months for the PRP group (mean - 4.6 $\pm 1.6 ;-77 \%)$ and at 1 month in the CS group $(-3.4 \pm$ $1.2 ;-58 \%)$.

The pharmacological effect of CS seemed to disappear 15 weeks after receiving treatment as all scores tended to worsen after this period. For instance, pain in the CS group improved rapidly but, in general, worsened after 15 weeks of treatment, and the pain steadily increased in each follow-up visit. At the same time, the PRP group resulted in a sustained improvement in pain relief up to 30 weeks, showing a small increase in pain in the 1 -year evaluation follow-up (Fig. 2a). For all other outcome scores, there were significant differences between pretreatment and post-treatment results at any time, evaluated up to 58 weeks of the follow-up $(p<0.05)$, except for the VAS $(p=0.1537)$ and KSS $(p=0.1719)$ indexes for the CS group at 58 weeks (due to worsening of the pain conditions of the patients).

Knee function improvement was observed in both groups up to 5-15 weeks with no significant differences between groups $(p>0.05)$ (Table 2). At V4 (15 weeks), the PRP group presented a better significant improvement in the IKDC and KSS scores compared to the CS group, which decreased in effectiveness up to 1 year (Fig. 2b, c). Maximum functional improvement and better patient expectation, satisfaction, and activity levels were observed after 15 weeks for the PRP group (mean change from baseline of $41.1 \pm 13.6,112 \%$ and $30.2 \pm$ $11.7,51 \%$ for IKDC) and after 5 weeks for the CS group (mean change from baseline of $33.7 \pm 13.5,111 \%$ and $29.4 \pm 12.8,55 \%$ for KSS).

\section{Safety}

No serious adverse events (SAE) occurred. No adverse events were registered in the CS group. Mild synovitis was registered by 15 patients $(75 \%)$ in the PRP group at the first week after treatment (diagnosed by ultrasound and clinical evaluation: patellar tap test, brush test, fluid displacement, and wave test) that resolved spontaneously. No synovitis was reported from the patients of the CS group.

\section{Discussion}

This single-center prospective randomized controlled study showed that a single intra-articular injection of PRP was more efficient than CS for treating moderate OA (Kellgren-Lawrence grades II-III) compared to triamcinolone acetonide. The effectiveness of PRP has been questioned by some authors because the evidence of its efficacy has been highly variable depending on the specific indication [21-24]. Other studies have shown that PRP has been effective for knee OA when compared to placebo, ozone, or HA in several high-quality, randomized, controlled trials [25-29]. Some of these studies suggested that intra-articular infiltrations of PRP provide quantifiable benefits for pain relief and functional improvement within a limited time period (up to 1 year) [25, 28, 29]. For instance, Filardo et al. [30] performed three consecutive intra-articular infiltrations of PRP in a group of 91 patients with chronic degenerative knee 
Table 2 Primary outcome and secondary outcomes in per-protocol population during the follow-up of the study

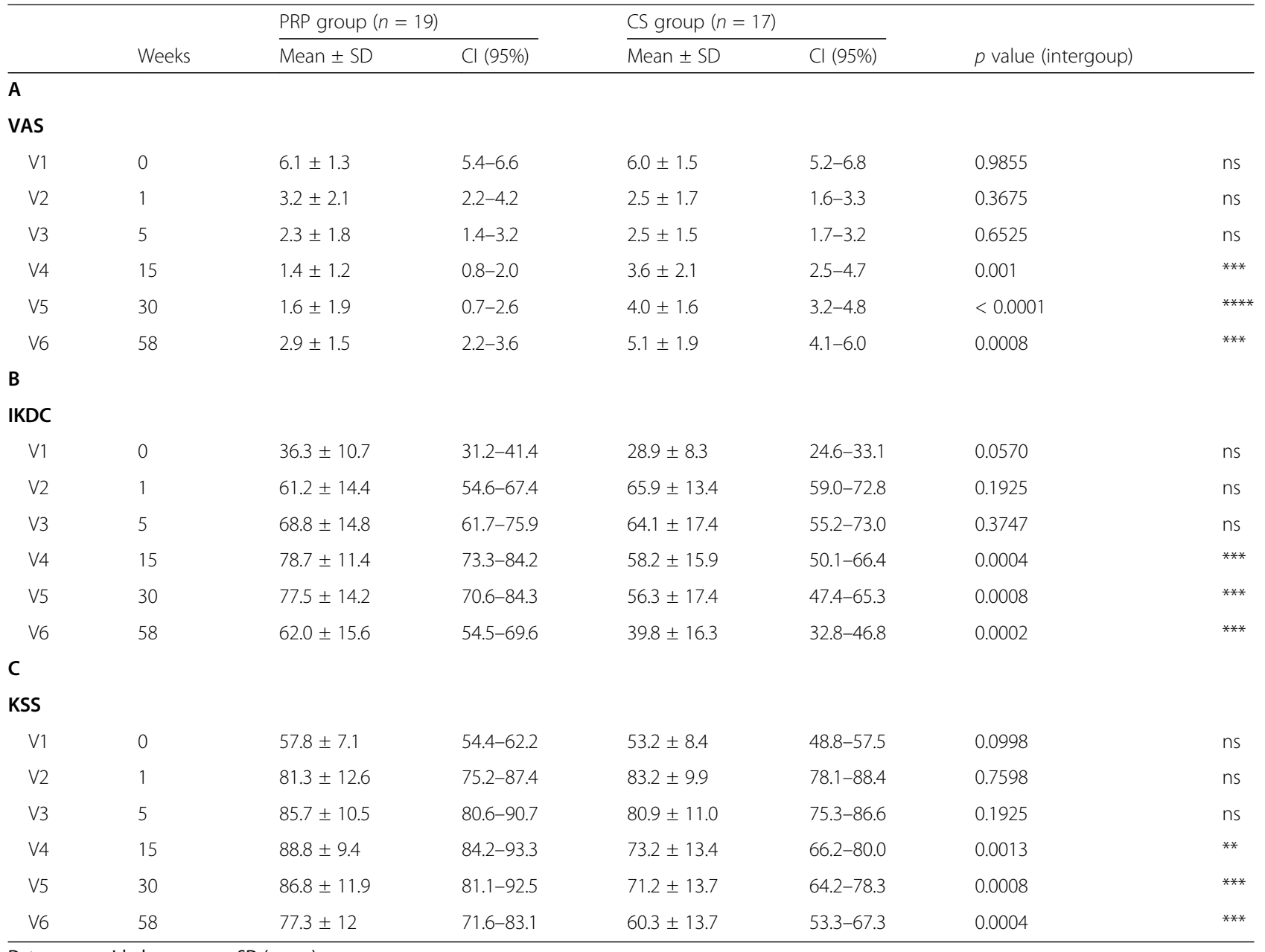

Data are provided as mean \pm SD (range)

$p$ value of Mann-Whitney test

ns not significant, VAS visual analog scale, KSS Knee Society Score, IKDC International Knee Documentation Committee, $C I$ confidence interval

** Statistically significant difference $(p \leq 0.01)$

*** Statistically significant difference $(p \leq 0.001)$

**** Statistically significant difference $(p \leq 0.0001)$

conditions with improvement in the IKDC and EQ-VAS indexes up to 1 year. However, this condition deteriorated 24 months after 1 year of clinical improvements (especially for younger patients with a low to mild degree of cartilage degeneration). Similar results were reported by Fukawa et at [31]., who performed three consecutive injections of PRP with a VAS reduction up to 1 year with a deterioration of the improvement after 24 months. In this study, a single intra-articular injection of PRP resulted in significant pain relief for up to 12 months, with a maximum pain decrease after 3 months. This difference suggested that the number of PRP injections could be critical for the maintenance of the beneficial effect. In contrast to this, Patel et al. [32] did not find significant differences in WOMAC scores between single and double PRP injections for early OA at 6 weeks and 3 and 6 months.
As far as we know, only a few trials have been published that compare clinical outcomes after PRP and corticosteroid (CS) injections for treating mild OA [16-18]. In general, those studies concluded that a single dose of PRP is comparable to 1 dose of CS (none of them analyzed the very short-term effects-1 week). Most of the studies of PRP commonly perform the first assessment of patients 1 month after treatment, making impossible the determination of the evolution in the first few days [33]. Based on this remark and based on our personal experience that we have from our routine care activity, we evaluated the clinical outcome of the patients in the very short term (1 week). From our knowledge, this is the first PRP clinical study for early-stage knee OA that incorporated outcome assessment by means of this approach. We found that the analgesic effect of PRP in 


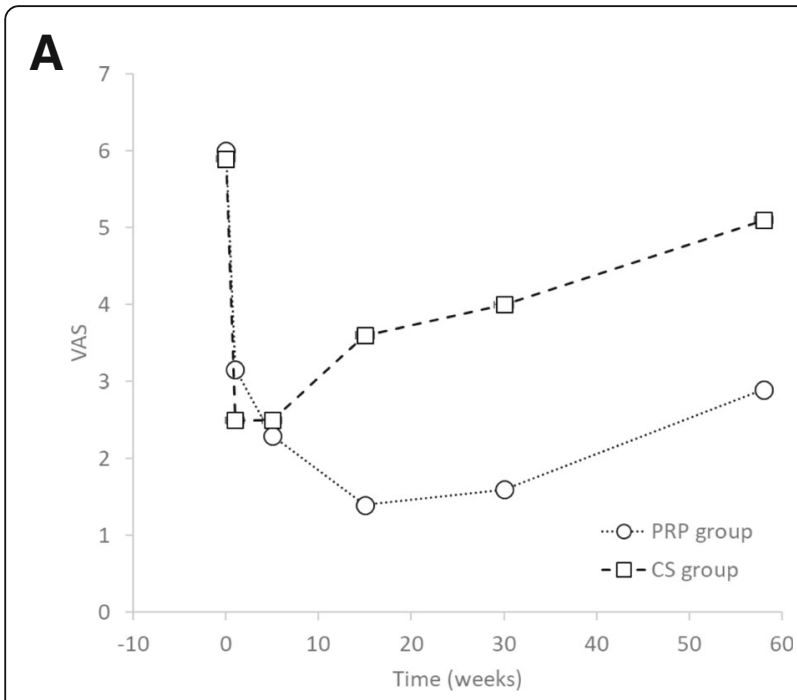

B

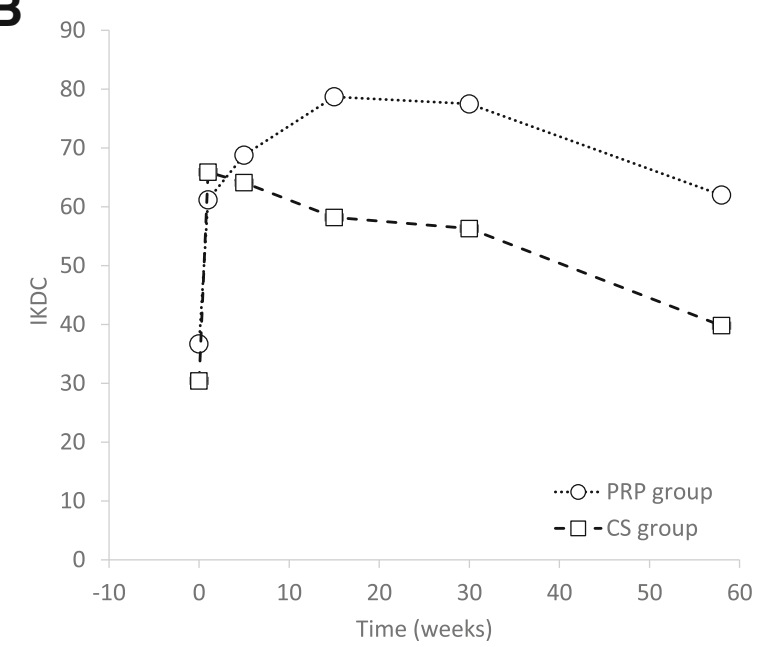

C

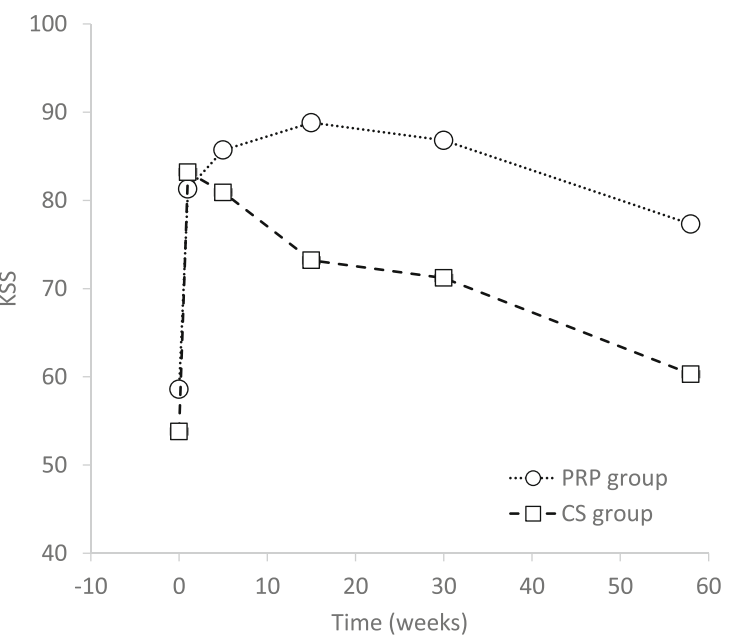

Fig. 2 Evaluation of the subjective state according to VAS (a), IKDC (b), and KSS (c) over time. Values are the average of each score at any one time. VAS, visual analog scale; KSS, Knee Society Score; IKDC, International Knee Documentation Committee the very short term was comparable to CS, and the same was noticed for the knee function (Table 2; 1 to 5 weeks; ns). The rapid reduction in pain observed upon treatment with PRP might be attributed to a combined effect, mainly due to the induction of endogenous cell endocannabinoids [34] together with the anti-inflammatory activity effect of PRP on chondrocytes [35, 36]. The variation in pain and knee function in the PRP group contributed to the sustained duration of the overall beneficial effects for up to 30 weeks, whereas in the CS group, a tendency to worsen after 5 weeks was registered. A recent metaanalysis reported the limitation of the beneficial effect of the CS 15 weeks after the end of treatment [37]. We hypothesized that the improvement of both parameters (pain and function) were mainly due to control of the inflammation of the knee rather than the trophic effect of PRP on cartilage. This rationale is due to the fact that it has not yet been demonstrated that the improvement of knee function after PRP treatment correlates with a volume increase of the articular cartilage [38-40], even when has been demonstrated that in vitro, TGF- $\beta 1 / 3$ can induce chondrogenesis of mesenchymal stem cells [41]. However, there is evidence that suggests that PRP has other effects on the joints other than the anti-inflammatory and this may probably explain why the group that received PRP had better results than the CS group. Even though the mechanism of action on improving cartilage repair remains unclear, it has been reported in the literature that PRP can induce tissue maturation characterized by increased cell proliferation and tissue stiffness [42]. These cells, in turn, produce more superficial zone protein that functions as a boundary lubricant that helps reduce friction and wear [43, 44]. Moreover, it has been reported that PRP can enhance HA secretion from synovial fibroblasts in arthritic patients, producing a lubricating effect that could reduce the shear stress of the joint [45].

Administration of CS for treating OA has been controversial because these injections can reduce pain in the short term, but they may not be helpful in the treatment of the underlying arthritic lesion [46]. CS administrations have been reported to have deleterious effects on musculoskeletal tissues such as reduction of collagen synthesis, suppression of cell proliferation, induction of oxidative stress, and impact on cell viability [47-50]. This harmful effect could also influence the difference in long-term results between treatment groups in detriment of the CS group.

Intra-articular PRP infiltrations have been widely used for the treatment of knee OA with many beneficial results $[28,32,51]$. In this study, intra-articular PRP injections were well tolerated. The most common side effect being mild synovitis tended to resolve within the first 
week after treatment. Treatment with PRP injections can be considered safe since no severe adverse events or complications have been reported. We consider that the safety of PRP is mainly due to two factors: the administration of the PRP done through minimally invasive procedures and non-existent risk of transmission of infectious diseases (because it is an autologous procedure). In addition, in this study, we used pure PRP (the leukocyte layer fraction was not recovered per manufacturer's instructions) to prevent possible inflammatory reactions [52, 53] in spite of the reported safety of using leucocyte-rich PRP [54]. Another relevant aspect of this study was that we plotted our results on a numerical scale (Fig. 2). Curiously, most of the studies represent the time variable on non-numerical plots which masked the real trends. This makes it difficult to appreciate the rate of the clinical improvement or worsening. For instance, outcome evolution graphs with ordinate axes $(X)$ with equidistant values of 1,3 , and 6 months are frequently found in the literature $[38,55,56]$.

\section{Limitations}

The major limitation of the study was the absence of double-blinding. Blinding was not possible for the patients because the PRP preparation requires an additional blood withdrawal that would not be required for the CS group. Blinding was not possible for the clinicians who performed the infiltration because of the visible different aspects of the two compounds. Also, the number of patients included in this trial might have been underestimated. Although a sample size calculation was performed based on the assumption of an expected improvement of 1.5 points in VAS pain in the treatment group vs the control group at 1 year after treatment, and given that pain is a very subjective variable, the sample might need to be larger. Moreover, the loss of 4 patients due to causes not related to the treatment ( 1 for the PRP group and 3 for the CS group) could be relevant for the analysis of the clinical results. Other limitations were the absence of magnetic resonance imaging (MRI) data which is an important objective measurement to determine the potential effect of PRP on the cartilage tissue. Another limitation was that the baseline IKDC scores showed statistically significant differences between groups, maybe due to due to the low sample size and that could induce bias in the analysis of the results.

\section{Conclusion}

This study shows that one intra-articular PRP injection is safe, it can reduce pain, and it can improve the knee function of patients with mild/moderate knee OA. The PRP intra-articular injection improves short-term scores of pain and knee function with no significant difference when compared to corticosteroids. However, PRP treatment resulted in a longer sustained effect than triamcinolone up to 12 months after administration. PRP can also produce a mild synovitis in the first week after administration without risk for the patient. It is recommended that a larger, randomized clinical trial should be performed to further assess the efficacy of PRP treatment in patients with mild/moderate OA.

\section{Abbreviations \\ BMI: Body mass index; Cs: Corticosteroid; IA: Intra-articular (IA); IKDC: International Knee Documentation Committee; ISRCTN: International Standard Randomized Controlled Trial Number; KSS: Knee Society Score; MRI: Magnetic resonance imaging; NSAIDs: Non-steroidal anti-inflammatory drugs; OA: Osteoarthritis; PRP: Platelet-rich plasma; SAE: Serious adverse events; TGF: Transforming growth factor; VAS: Visual analog scale}

\section{Acknowledgements}

We want to thank Marco A. Vegas-Landeau, Carlo Barbera, Irene Beriotto, and Nicola Giordan for carefully reading the manuscript.

\section{Authors' contributions}

AF and AP conceived, designed, and performed the trial. AF and AP analyzed the data. LV performed the statistical analysis. AF wrote the paper. The authors read and approved the final version of the manuscript.

\section{Funding}

No funding was received for this study.

Availability of data and materials

Data is available via the corresponding author under a reasonable request.

\section{Ethics approval and consent to participate}

The Medical Ethics Committee of Rigas Stradins University (RSU) and Ethics Committee of Medical and Biomedical Research Foundation of Riga Eastern Clinical University Hospital Support Foundation (Ref E-9(2))

\section{Consent for publication}

Not applicable

\section{Competing interests}

The authors declare that they have no competing interests. LV is the scientific affairs manager from FDIA Spain and gave support to the statistical analysis.

\section{Author details}

${ }^{1}$ Faculty of Continuing Education, Rīga Stradinš University, Riga, Latvia. 2"ORTO klinika" Ltd., Riga, Latvia. ³aboratorios Fidia Farmacéutica S.L.U, Madrid, Spain.

Received: 3 October 2019 Accepted: 16 June 2020

Published online: 10 July 2020

References

1. Creamer P, Hochberg MC. Osteoarthritis. Lancet. 1997;350(9076):503-8.

2. Sanchez M, Delgado D, Sanchez P, Fiz N, Azofra J, Orive G, et al. Platelet rich plasma and knee surgery. Biomed Res Int. 2014;2014:890630.

3. Onishi K, Utturkar A, Chang E, Panush R, Hata J, Perret-Karimi D. Osteoarthritis: a critical review. Crit Rev Phys Rehabil Med. 2012;24(3-4):251-64.

4. Lane NE, Shidara K, Wise BL. Osteoarthritis year in review 2016: clinical. Osteoarthr Cartil. 2017;25(2):209-15.

5. Bannuru RR, Osani MC, Vaysbrot EE, Arden NK, Bennell K, Bierma-Zeinstra SMA, et al. OARSI guidelines for the non-surgical management of knee, hip, and polyarticular osteoarthritis. Osteoarthr Cartil. 2019;27(11):1578-89.

6. Steinmeyer J, Bock F, Stove J, Jerosch J, Flechtenmacher J. Pharmacological treatment of knee osteoarthritis: special considerations of the new German guideline. Orthop Rev (Pavia). 2018;10(4):7782

7. Ayhan E, Kesmezacar H, Akgun I. Intraarticular injections (corticosteroid, hyaluronic acid, platelet rich plasma) for the knee osteoarthritis. World J Orthop. 2014;5(3):351-61. 
8. Wernecke C, Braun HJ, Dragoo JL. The effect of intra-articular corticosteroids on articular cartilage: a systematic review. Orthopaedic journal of sports medicine. 2015;3(5):2325967115581163.

9. Dragoo JL, Danial CM, Braun HJ, Pouliot MA, Kim HJ. The chondrotoxicity of single-dose corticosteroids. Knee surgery, sports traumatology, arthroscopy: official journal of the ESSKA. 2012;20(9):1809-14

10. Andia I, Maffulli N. Blood-derived products for tissue repair/regeneration. Int J Mol Sci. 2019;20(18):4581.

11. Andia I, Maffulli N. A contemporary view of platelet-rich plasma therapies: moving toward refined clinical protocols and precise indications. Regen Med. 2018;13(6):717.

12. Andia I, Maffulli N. How far have biological therapies come in regenerative sports medicine? Expert Opin Biol Ther. 2018;18(7):785.

13. Andia I, Maffulli N. Biological therapies in regenerative sports medicine. Sports medicine (Auckland, NZ). 2017;47(5):807.

14. Andia I, Sánchez M, Maffulli N. Joint pathology and platelet-rich plasma therapies. Expert Opin Biol Ther. 2012;12(1):7.

15. Andia I, Maffulli N. New biotechnologies for musculoskeletal injuries. Surgeon. 2019;17(4):244.

16. Forogh B, Mianehsaz E, Shoaee S, Ahadi T, Raissi GR, Sajadi S. Effect of single injection of platelet-rich plasma in comparison with corticosteroid on knee osteoarthritis: a double-blind randomized clinical trial. J Sports Med Phys Fitness. 2016:56(7-8):901-8.

17. Joshi Jubert N, Rodriguez L, Reverte-Vinaixa MM, Navarro A. Platelet-rich plasma injections for advanced knee osteoarthritis: a prospective, randomized, double-blinded clinical trial. Orthop J Sports Med. 2017;5(2): 2325967116689386

18. Huang Y, Liu X, Xu X, Liu J. Intra-articular injections of platelet-rich plasma, hyaluronic acid or corticosteroids for knee osteoarthritis: a prospective randomized controlled study. Orthopade. 2019;48(3):239-47.

19. Jorgensen TS, Graven-Nielsen T, Ellegaard K, Danneskiold-Samsoe B, Bliddal $H$, Henriksen M. Intra-articular analgesia and steroid reduce pain sensitivity in knee OA patients: an interventional cohort study. Pain Res Treat. 2014; 2014:710490.

20. Insall JN, Dorr LD, Scott RD, Scott WN. Rationale of the knee society clinical rating system. Clin Orthop Relat Res. 1989;248:13-4.

21. Scott A, LaPrade RF, Harmon KG, Filardo G, Kon E, Della Villa S, et al. Platelet-rich plasma for patellar tendinopathy: a randomized controlled trial of leukocyte-rich PRP or leukocyte-poor PRP versus saline. Am J Sports Med. 2019:47(7):1654-61.

22. de Jonge $S$, de Vos RJ, Weir A, van Schie HT, Bierma-Zeinstra SM, Verhaar JA, et al. One-year follow-up of platelet-rich plasma treatment in chronic Achilles tendinopathy: a double-blind randomized placebo-controlled trial. Am J Sports Med. 2011;39(8):1623-9.

23. Le ADK, Enweze L, DeBaun MR, Dragoo JL. Current clinical recommendations for use of platelet-rich plasma. Curr Rev Musculoskelet Med. 2018;11(4):624-34.

24. Filardo G, Di Matteo B, Kon E, Merli G, Marcacci M. Platelet-rich plasma in tendon-related disorders: results and indications. Knee Surg Sports Traumatol Arthrosc. 2018;26(7):1984-99

25. Shen $L$, Yuan $T$, Chen $S$, Xie X, Zhang $C$. The temporal effect of platelet-rich plasma on pain and physical function in the treatment of knee osteoarthritis: systematic review and meta-analysis of randomized controlled trials. J Orthop Surg Res. 2017;12(1):16.

26. Ye Y, Zhou X, Mao S, Zhang J, Lin B. Platelet rich plasma versus hyaluronic acid in patients with hip osteoarthritis: a meta-analysis of randomized controlled trials. Int J Surg. 2018:53:279-87.

27. Meheux CJ, McCulloch PC, Lintner DM, Varner KE, Harris JD. Efficacy of intraarticular platelet-rich plasma injections in knee osteoarthritis: a systematic review. Arthroscopy. 2016;32(3):495-505.

28. Dai WL, Zhou AG, Zhang H, Zhang J. Efficacy of platelet-rich plasma in the treatment of knee osteoarthritis: a meta-analysis of randomized controlled trials. Arthroscopy. 2017;33(3):659-70.e1.

29. Laudy AB, Bakker EW, Rekers M, Moen MH. Efficacy of platelet-rich plasma injections in osteoarthritis of the knee: a systematic review and metaanalysis. Br J Sports Med. 2015;49(10):657-72.

30. Filardo G, Kon E, Buda R, Timoncini A, Di Martino A, Cenacchi A, et al. Platelet-rich plasma intra-articular knee injections for the treatment of degenerative cartilage lesions and osteoarthritis. Knee Surg Sports Traumatol Arthrosc. 2011:19(4):528-35.
31. Fukawa T, Yamaguchi S, Akatsu Y, Yamamoto Y, Akagi R, Sasho T. Safety and efficacy of intra-articular injection of platelet-rich plasma in patients with ankle osteoarthritis. Foot Ankle Int. 2017;38(6):596-604.

32. Patel S, Dhillon MS, Aggarwal S, Marwaha N, Jain A. Treatment with platelet-rich plasma is more effective than placebo for knee osteoarthritis: a prospective, double-blind, randomized trial. Am J Sports Med. 2013;41(2):356-64.

33. Cole BJ, Karas V, Hussey K, Pilz K, Fortier LA. Hyaluronic acid versus plateletrich plasma: a prospective, double-blind randomized controlled trial comparing clinical outcomes and effects on intra-articular biology for the treatment of knee osteoarthritis. Am J Sports Med. 2017;45(2):339-46.

34. Descalzi F, Ulivi V, Cancedda R, Piscitelli F, Luongo L, Guida F, et al. Plateletrich plasma exerts antinociceptive activity by a peripheral endocannabinoidrelated mechanism. Tissue Eng Part A. 2013;19(19-20):2120-9.

35. Andia I, Maffulli N. Platelet-rich plasma for managing pain and inflammation in osteoarthritis. Nat Rev Rheumatol. 2013:9(12):721-30.

36. Moussa M, Lajeunesse D, Hilal G, El Atat O, Haykal G, Serhal R, et al. Platelet rich plasma (PRP) induces chondroprotection via increasing autophagy, anti-inflammatory markers, and decreasing apoptosis in human osteoarthritic cartilage. Exp Cell Res. 2017:352(1):146-56.

37. Juni P, Hari R, Rutjes AW, Fischer R, Silletta MG, Reichenbach S, et al. Intraarticular corticosteroid for knee osteoarthritis. Cochrane Database Syst Rev. 2015;(10):Cd005328.

38. Guillibert C, Charpin C, Raffray M, Benmenni A, Dehaut F-X, El Ghobeira G et al. Single injection of high volume of autologous pure PRP provides a significant improvement in knee osteoarthritis: a prospective routine care study. Int J Mol Sci. 2019;20(6):1327.

39. Buendia-Lopez D, Medina-Quiros M, Fernandez-Villacanas Marin MA. Clinical and radiographic comparison of a single LP-PRP injection, a single hyaluronic acid injection and daily NSAID administration with a 52-week follow-up: a randomized controlled trial. J Orthop Traumatol. 2018;19(1):3.

40. Burchard R, Huflage H, Soost C, Richter O, Bouillon B, Graw JA. Efficiency of platelet-rich plasma therapy in knee osteoarthritis does not depend on level of cartilage damage. J Orthop Surg Res. 2019;14(1):153.

41. Goldberg A, Mitchell K, Soans J, Kim L, Zaidi R. The use of mesenchymal stem cells for cartilage repair and regeneration: a systematic review. J Orthop Surg Res. 2017;12(1):39.

42. Zhang Y, Morgan BJ, Smith R, Fellows CR, Thornton C, Snow M, et al. Platelet-rich plasma induces post-natal maturation of immature articular cartilage and correlates with LOXL1 activation. Sci Rep. 2017, 7:(1):3699.

43. Sakata R, McNary SM, Miyatake K, Lee CA, Van den Bogaerde JM, Marder RA, et al. Stimulation of the superficial zone protein and lubrication in the articular cartilage by human platelet-rich plasma. Am J Sports Med. 2015; 43(6):1467-73.

44. Sakata R, Reddi AH. Platelet-rich plasma modulates actions on articular cartilage lubrication and regeneration. Tissue Eng Part B Rev. 2016;22(5): 408-19.

45. Anitua E, Sanchez M, Nurden AT, Zalduendo MM, de la Fuente M, Azofra J, et al. Platelet-released growth factors enhance the secretion of hyaluronic acid and induce hepatocyte growth factor production by synovial fibroblasts from arthritic patients. Rheumatology (Oxford, England). 2007; 46(12):1769-72.

46. Bellamy N, Campbell J, Robinson V, Gee T, Bourne R, Wells G. Intraarticular corticosteroid for treatment of osteoarthritis of the knee. Cochrane Database Syst Rev. 2006;19(2):Cd005328.

47. Stove J, Schoniger R, Huch K, Brenner R, Gunther KP, Puhl W, et al. Effects of dexamethasone on proteoglycan content and gene expression of IL-1 betastimulated osteoarthrotic chondrocytes in vitro. Acta Orthop Scand. 2002; 73(5):562-7.

48. Xue E, Zhang Y, Song B, Xiao J, Shi Z. Effect of autophagy induced by dexamethasone on senescence in chondrocytes. Mol Med Rep. 2016;14(4): 3037-44.

49. Muto T, Kokubu T, Mifune Y, Inui A, Sakata R, Harada Y, et al. Effects of plateletrich plasma and triamcinolone acetonide on interleukin-1ss-stimulated human rotator cuff-derived cells. Bone Joint Res. 2016:5(12):602-9.

50. Suntiparpluacha M, Tammachote N, Tammachote R. Triamcinolone acetonide reduces viability, induces oxidative stress, and alters gene expressions of human chondrocytes. Eur Rev Med Pharmacol Sci. 2016; 20(23):4985-92.

51. Smith PA. Intra-articular autologous conditioned plasma injections provide safe and efficacious treatment for knee osteoarthritis: an FDA-sanctioned, 
randomized, double-blind, placebo-controlled clinical trial. Am J Sports Med. 2016;44(4):884-91.

52. Braun HJ, Kim HJ, Chu CR, Dragoo JL. The effect of platelet-rich plasma formulations and blood products on human synoviocytes: implications for intra-articular injury and therapy. Am J Sports Med. 2014;42(5):1204-10.

53. Xu Z, Yin W, Zhang Y, Qi X, Chen Y, Xie X, et al. Comparative evaluation of leukocyte- and platelet-rich plasma and pure platelet-rich plasma for cartilage regeneration. Sci Rep. 2017;7:43301.

54. Riboh JC, Saltzman BM, Yanke AB, Fortier L, Cole BJ. Effect of leukocyte concentration on the efficacy of platelet-rich plasma in the treatment of knee osteoarthritis. Am J Sports Med. 2016;44(3):792-800.

55. Louis ML, Magalon J, Jouve E, Bornet CE, Mattei JC, Chagnaud C, et al. Arthroscopy. 2018;34(5):1530-40.e2

56. Su K, Bai Y, Wang J, Zhang H, Liu H, Ma S. Comparison of hyaluronic acid and PRP intra-articular injection with combined intra-articular and intraosseous PRP injections to treat patients with knee osteoarthritis. Clin Rheumatol. 2018;37(5):1341-50.

\section{Publisher's Note}

Springer Nature remains neutral with regard to jurisdictional claims in published maps and institutional affiliations.

\section{Ready to submit your research? Choose BMC and benefit from:}

- fast, convenient online submission

- thorough peer review by experienced researchers in your field

- rapid publication on acceptance

- support for research data, including large and complex data types

- gold Open Access which fosters wider collaboration and increased citations

- maximum visibility for your research: over $100 \mathrm{M}$ website views per year

At $\mathrm{BMC}$, research is always in progress.

Learn more biomedcentral.com/submissions 